\title{
CRIMSONpublishers
}

\author{
http://www.crimsonpublishers.com
}

\section{Aged Care Physiotherapy: Are We Doing it Right?}

\author{
Nitish Mathew* \\ Consultant Physiotherapist, Revita Health for seniors, Australia \\ *Corresponding author: Nitish Mathew, Consultant Physiotherapist, Revita Health for seniors, Australia

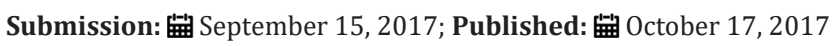

\section{Introduction}

Per the most recent census in 2016, there are more than 3.2 million Australians between 65-84 years of age. There are, further, 487,000 people aged over 85 years of age [1]. In recent years there has been a tremendous growth in the Australian population and also a decrease in the number of deaths secondary related to major conditions like cancer, COPD, CVD, etc. The Australian Bureau of statistics predicts the total number of Australians over the age of 65 years will increase to more than 6 million by 2051. It is estimated that by 2051, around 3.5 million people in the older age group will be using aged care system [2]. The Negative effects of ageing include changes in muscle strength, joint cartilage, bone density and body fat; these changes, leads to poor fitness, thereby causing further health complications such as dyspnea, hypertension, arthritis, circulatory issues, stroke, etc. [3]. Another common health issue in the aged population is psychological disorders which affect cognition and orientation, such as Dementia, delirium.

Geriatric (Aged care) Physiotherapy was introduced as a specialty in physiotherapy in 1989. Since then, physiotherapists have worked together to discover and understand those age related problems which can be treated or managed through physiotherapy. Physiotherapists play as major role primary care practitioners in treating and managing the functional limitations aged patients that can result from common conditions like a stroke, Parkinson's disease, arthritis, COPD, cancer or joint replacement surgeries. Physiotherapists check the suitability of particular modalities or interventions for the treatment of musculoskeletal, neurovascular and cardiopulmonary conditions. Their role is important in patient rehabilitation after a fall, for injury prevention, and for functional incontinence [4]. The benefits of geriatric physiotherapy in improving mobility, balance, strength, and confidence in performing the activities of daily life and the ability to live actively life for longer, have been proven worldwide.

\section{Duties of Geriatric Physiotherapist}

A geriatric physiotherapist is, in some ways, a jack of all trades. Geriatric physiotherapists should have sound knowledge of respiratory care, orthopedics, medicine and neurology; they should also have a firm grasp on the psychosocial aspects of medical care, to provide a holistic and client-centered care.
General principles practiced geriatric physiotherapists include [5]:

i. Find the root cause of disability, i.e. the pathological cause/ injury, which makes the clients dependent on others.

ii. Maintain the functioning of the patient's body systems to their optimum levels enhancing mobility and independence.

iii. Enable a pain-free and comfortable life for all clients.

iv. Promote health by preventing future health problems.

The geriatric physiotherapist should focus on the patient's goals while planning a treatment protocol. At the same time, the treatment should center management and improvement of the health condition. Often, it is complete management and improvement of the health of the client is not possible. On these occasions, the physiotherapist can focus on the chief complaints of their client, thereby providing pain relief and care for them. Reversing the health problems of the clients and returning them to earlier states of health are not always possible, but the key is to help clients function to the best of their abilities.

Understanding the problems of clients suffering from brain disorders, such as dementia, is a complex task. People with dementia face difficulty in expressing the nature and site of their pain. They may have episodes of depression or agitation as a result of their health problems. Pain in these clients also affects cognition, concentration, and motivation for any therapeutic intervention [6]. Geriatric physiotherapists, with their effective communication skills and specialized knowledge, can diagnose the sites of pain and provide appropriate management to improve the quality of life of these clients. They can provide proper family and caregiver education, to support and manage the lifestyle of aged people with dementia.

Falls are a common problem for elderly people due to the neurological, skeletal and visual disorders that often affect aged patients [7]. A well-maintained exercise program can reduce the chances of falls in aged clients by 54 percent [8]. Therapists need to assess the root cause of each fall, which is either extrinsic or intrinsic and come up with appropriate solutions to prevent them in the future. Hence, geriatric physiotherapists witness the same signs and symptoms in their patients, which are observed in other streams 
of physiotherapy. A geriatric physiotherapist needs to follow the same assessment and treatment protocols which they would use with other populations. A significant problem, however, is that the elderly generation is unable to get access to regular physiotherapy in Australia. This is due to a number of complications. The major reason is there are only a small number of physiotherapists willing to work in the aged care sector. The poor funding provided by Medicare and ACFI towards physiotherapy programs is another common barrier [9]. People living in rural areas also suffer because of the smaller number of centers providing physiotherapy, and other barriers such as lack of access to transport, long travel distances, and complications from existing disabilities [10].

Barriers in Aged Care Physiotherapy

A. Disinterest in working in the aged care sector among physiotherapists:

i. Physiotherapists feel that the workload in aged care is too much, too stressful and too boring.

ii. Physiotherapists feel there is less professional development in this field.

iii. Physiotherapy assistants have less knowledge and experience, causing workload increases and leading to decreased job satisfaction [11].

iv. The average salary is lower than in other streams of physiotherapy.

\section{B. Government rules:}

i. There is no national framework stating the amount of physiotherapy care, in terms of duration, to be provided for patients in the aged care sector.

C. Funds are inadequate:

i. The major funding providers, like the Aged Care Funding Instrument, grant no funds for rehabilitation of functional independence, falls prevention, or strength training.

ii. The Medicare Chronic Disease Management Program limits claimants to only 5 allied health services in one year, and those services are decided by the general practitioner.

iii. The funding providers, like Rural Health Workforce Australia, who used to allocate funds and reimbursements for therapists willing to work in more rural areas, do not have such practices at present, due to a change in laws [12].

\section{Aged Care Funding Instrument}

i. $\quad$ The Aged Care Funding Instrument provides financial grants for treatment of residents in aged care homes. It focuses on passive pain management protocols and offers nothing for active functional training for independence.

ii. Physiotherapy treatments according to the ACFI include pain management with the help of therapeutic massage or electrotherapeutic modalities, which can be given to the residents at least once and up to 4 times per week. Duration is set at 20 minutes per session according to the 2017 regulations.

iii. Physiotherapists decide the frequency of treatments (from one to 4 times per week) which should be then approved by ACFI.

iv. The present model is prescriptive, and is not based on clinical assessments or need; as such, there is little, evidence that it will improve the quality of life of residents.

Many physiotherapy organizations and therapists have come forward with their views on the drawbacks of this tool. Rik Dawson, Gerontology Chief of the; APA, appreciates the focus on pain management in the funds allocated to residents but points out the failure in not including exercises or evidence-based practices, like Cognitive Behavioral Therapy, joint mobilization, and postural correction, as part of pain management. He adds that the evidence of the benefits of exercise for older people with arthritis are greater than the evidence for massage or TENS [13].

Jennifer Hewitt, an APA member, adds that frequent falls inside aged care centers form a major factor in the increasing pain in the residents. The cost of falls in these residences is more than $20 \%$ of the total cost of falls for the entire healthcare system. Prevention of falls should be a priority in these homes in order to maintain the health of residents, minimizing pain and maximizing independence and mobility. Hewitt proved the benefits of exercise over pain as well as prevention of falls.

Anita Powell, the executive officer of ESSA, is disappointed that exercise physiology is not included in the ACFI tool, and that, as a result, aged care centers do not appoint exercise physiologists. Apart from the strength and pain management benefits of exercise for the older population, she also points out the psychological benefits of exercises for this population. She offers evidence of increased socialization among isolated residents, of improved happiness and health of residents, and also of engaged interest and fun for the residents during group exercise programs [14]. At the same time, there have been public comments on media stating that ACFI allows unethical practices. Families of residents in nursing homes have reported their concern that aged care providers keep searching for ways to increase their funding, but without offering evidence of relevant, improved outcomes for the residents (Aged care crisis Inc., 2015). APA has submitted a request to the government to reform the ACFI and to allow care center residents to benefit from physiotherapy-managed exercise to reduce pain and increase independence and quality of life (APA, 2010).

For these reasons, many physiotherapists in Australia refuse to work in aged care. To solve this problem, Australian employers hire a number of overseas physiotherapists on sponsorship work visas. In 2012, 3,006 out of 17,075 employed physiotherapists were from countries other than Australia. The number of therapists hired on 457 sponsorship work visas increased from 90 in 2009 to 118 in 2013. Similarly, therapists hired through employer-sponsored permanent visas or general skilled migration visas appear in higher numbers each year [15]. Physiotherapy has suffered from a skills shortage for many years, but a majority of the new therapists join 
streams other than aged care, which puts aged care residential and nursing homes in crisis. Residential homes in Australia hire physiotherapists part-time: for only one day per week or for an on-call basis. The high number of fresh graduate physiotherapists (with no relevant working experience) and occupational therapists working in the field of aged care is also due to the same reason of low number of physiotherapists interested in this field.

\section{Benefits of Work as a Geriatric Physiotherapist}

Compared to the other streams of physiotherapy, aged care has fewer challenges for the practitioner and a smaller workload. Although residents in residential and nursing homes have various disorders and disabilities, the duty of the physiotherapist here is clearly focused on pain management as a primary concern. In a nutshell, the basic duties expected from the geriatric physiotherapist in Australia are [16]:

i. Pain management

ii. Reassessment and evaluation of interventions prescribed for residents

iii. Use of evidence-based assessment tools

iv. Record maintenance for the treatment hours for each resident

v. Assurance that residents' sessions are accurately filed and claimed through government associations.

For nursing homes which run on funding from the government, as all such funding agencies pay only for pain management through massage or TENS, the role of the physiotherapist is confined to this basic management. The physiotherapist alone determines whether to include exercise management as an addition in the pain management program. Hence, full-time physiotherapists in aged care are given full freedom to schedule their management as required, provided they follow the basic guidelines provided by ACFI for pain management. Another benefit of work as a geriatric physiotherapist is the salary. The average salary is $\$ 92,181$ annually, i.e. $\$ 43 /$ hour, which is 1.5 times more than the median wage of Australia. New graduates can earn around $\$ 65,000$, and experienced workers can earn over $\$ 129,000$ [17].

The increase in the geriatric population has added to the working hours of geriatric physiotherapists. Typically, these physiotherapists spend $46 \%$ of their time on pain management treatments, $25 \%$ on non- pain management treatments and $29 \%$ on documentation, as per a recent survey by Australian Physiotherapy Association [18]. Apart from all this, physiotherapists can bring joy to aged care center residents who are suffering from pain and feelings of dependence. Spending time with them, even for a friendly conversation for a few minutes, makes a lot of difference in their lives. Such time can also create a better trust relationship with clients, when they understand there is somebody to look after their health problems, somebody to go to if they are in pain.

Of course, a therapist in an aged care facility can limit their work to treating the residents for pain through massage, but they can make their work more worthwhile by including group exercise programs, balance classes, games sessions, and walks with residents, or use of stationary bikes or Swiss balls. Such exercises can be used as a treatment plan for improving the mobility, strength, and balance and gait of the residents. Many therapists who work full time in such facilities already practice the latter and have a healthy and happy work-life, while providing an active quality of life for the residents as well. A survey by APA shows that $60 \%$ of physiotherapists believe that the consultation and treatment time is not adequate. Physiotherapists have already joined hands all over Australia to increase their work hours.

There are 5,710 aged care residential homes in Australia. The older population will continue to grow. In 2056, the proportion of older Australians is expected to increase up to 8.7 million. By 2096, there will be more than 12.8 million Australians aged 65 years and above (AIHW, 2017). Australians are living longer, and medical standards are getting better and better, but are we really preserving and improving age with medicines? The value of geriatric physiotherapy is functionally universally recognized, but few people are available in Australia to support this ageing population. If spreading love and increasing smiles is your motto as a Physiotherapist, think again, these elderly champions are waiting for a guiding hand and a piece of motivation from you.

\section{References}

1. Australian Institute of health and welfare (2017). Australia's changing age and gender profile.

2. Australian Physiotherapy Association (2012) Supporting older Australians. Australian Physiotherapy Association.

3. Maguire A (2016) Physiotherapy for elderly patients; How it works: Balls bridge Physiotherapy clinic.

4. Aged care crisis Inc (2015) Aged Care finding Instrument (ACFI).

5. Ramaswamy B (2015) Physiotherapy and older people.

6. Chartered Society of Physiotherapy (2017) Physiotherapy works: Dementia care: Dementia action alliance.

7. Blood-Smyth J (2016) Physiotherapy for the elderly: Local Physio.

8. Falls Action (2015) Minimizing Falls: Falls screen.

9. Australian Physiotherapy Association (2010) Submission to the caring for older Australians inquiry. Productivity Commission 3-11.

10. Physiotherapists Aged care (2016) The growing rural population. PCare.

11. Higgins C (2012) Physiotherapy Assistants and the APA. Australian Physiotherapy Association.

12. My Health career (2016) Addressing Physiotherapy workforce shortages in rural and remote Australia. My health career.

13. Egan N (2016) Call for ACFI overhaul to cover exercise therapy for pain management. Australian Ageing Agenda.

14. Australian Ageing Agenda (2015) A new call for Allied health in aged care. Australian Ageing Agenda.

15. Human Workforce Australia (2014) Workforce inflows. Australia's health workforce series- Physiotherapists in focus 30- 33.

16. Wellness and Lifestyle Aged care services (2017) 8 things your aged care Physiotherapist should be doing. Physiotherapy articles.

17. Neuvoo (2017) Aged care physiotherapist salary in Australia.

18. Australian Physiotherapy Association (2014) ACFI Survey Australian Physiotherapy Association 6-14. 